\title{
Computer Aided Diagnosis for Virtual Colonography
}

\author{
Gabriel Kiss, Johan Van Cleynenbreugel, Maarten Thomeer, \\ Paul Suetens, Guy Marchal \\ Faculties of Medicine \& Engineering \\ Medical Image Computing (Radiology - ESAT/PSI) \\ University Hospital Gasthuisberg \\ Herestraat 49 \\ B-3000 Leuven, BELGIUM \\ Gabriel.Kiss@uz.kuleuven.ac.be
}

\begin{abstract}
The success of CT colonography (CTC) depends on appropriate tools for quick and accurate diagnostic reading. Current advancements in computer technology have the potential to bring such tools even to the PC level. In this paper a technique for Computed Aided Diagnosis (CAD) using CT colonography is described. The method labels positions in the volume data, which have a strong likelihood of being polyps and presents them in a userfriendly way. This method will reduce the amount of time needed by the radiologist to make a correct diagnosis. The method was tested on a study group of 18 patients and the sensitivity for polyps of $10 \mathrm{~mm}$ or larger was $100 \%$, comparable to that of human readers. The price paid for a high detection rate was a large number of approximately 8 false positive findings per case.
\end{abstract}

\section{Introduction}

Computed Tomographic Colonography (CTC) is a new non-invasive imaging tool that employs advanced imaging software to CT data for producing both two and three dimensional images of the colon [1]. The resulting images can be presented to a radiologist under one of the following formats: 2D axial slices, multi-planar reformatted 3D images (MPR), 3D endoluminal images or axial 3D images. The images can be generated instantaneously using software or hardware renderers or can be stored as digital movies.

The success of CTC depends on the applicability of the technique for large population screenings. The main limitations of CTC at this moment are the lengthy interpretation time and the possibility of perceptual errors.

Our experience [2] shows that the evaluation of 2D axial slices and axial 3D movies for both forward and reverse directions in both supine and prone positions takes about 30 minutes. The cost of CTC should be lower than the cost of colonography in order to be usable for large-scale screening. One possibility for time saving, and hence for cost saving is Computer Aided Diagnosis (CAD). Using CAD the radiologist does not have to concentrate on the whole volume, instead his attention will be focused only on small sub-volumes.

W. Niessen and M. Viergever (Eds.): MICCAI 2001, LNCS 2208, pp. 621-628, 2001.

(C) Springer-Verlag Berlin Heidelberg 2001 


\section{Short History of CAD}

Probably the best-known application area for CAD is mammography on digital highresolution radiographs [3]. With this technique breast calcifications are detected and classified, in order to prevent unnecessary surgical biopsies. Another area of applicability for CAD is the detection of lung cancer in CT images [4].

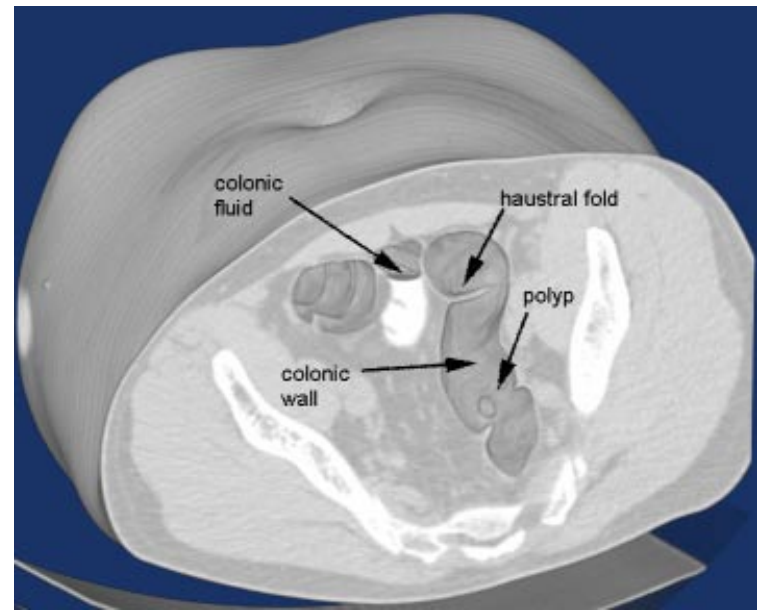

$\mathrm{CAD}$ for $\mathrm{CTC}$ is a relatively new research topic. The colon has a spatial extended and very complex structure (Figure 1), including smooth colonic wall, haustral folds, residual fluid and polyps. A direct implication of this fact is the huge amount of data that has to be inspected, resulting in timeconsuming CAD algorithms. Only recent advances in computing speed make these algorithms usable.

Fig. 1. The complex nature of the human colon.

Some approaches have been already applied in CTC CAD [5][6][7][8]; the methods used can be based on surface curvature, on surface normal, on sphere fitting, on polypoid shape detection, or on graph methods. Summers et al. tried to apply methods form virtual bronchoscopy to solve the CAD problem [9].

Surface curvature methods are based on concepts from differential geometry: minimum and maximum principal curvatures, mean and Gaussian curvatures. A thresholding based on these curvature values is applied and the remaining regions are declared as polyp candidates.

Surface normal methods exploit the fact that normals to the colonic surface intersect with neighboring normals depending on the curvature features of the colon. The main problem is the differentiation between polyps and haustral folds, which have similar curvature properties; one observation usable in this case is that haustral folds have a lower tendency of surface normal convergence than polyps, due to their cylindrical nature.

Sphere fitting methods exploit the spherical nature of polyps. Polyps can not be modeled as simple spheres because usually they are more complex entities. An alternative is to model polyps as spherical patches, followed by the detection of sphere center clusters situated in close vicinity (applying graph methods). 


\section{Method}

Our method uses a combination of the surface normal and sphere fitting methods and has the following steps: segmentation, polyp candidate generation, polyp center generation, polyp extraction and finally presentation. We preferred this alternative to an approach based on differential geometry due to its simplicity and computational efficiency.

To make the text easier to understand we start by presenting all of the threshold and predefined parameters used by our method. We also present their meaning and specify in which step they will appear. A visual summary of the method is shown in Figure 3.

\begin{tabular}{|l|l|l|l|}
\hline $\begin{array}{l}\text { Threshold } \\
\text { parameters }\end{array}$ & $\begin{array}{l}\text { Threshold } \\
\text { symbol }\end{array}$ & Step & Short description \\
\hline $\begin{array}{l}\text { Colonic } \\
\text { wall }\end{array}$ & $\mathrm{T}_{\text {segment }}$ & Segmentation & $\begin{array}{l}\text { Differentiate between air, colonic wall } \\
\text { and surrounding tissue }\end{array}$ \\
\hline $\begin{array}{l}\text { Bounding } \\
\text { box size }\end{array}$ & $\mathrm{T}_{\text {box }}$ & $\begin{array}{l}\text { Polyp } \\
\text { candidate } \\
\text { generation }\end{array}$ & $\begin{array}{l}\text { Defines the 3D volume of interest we } \\
\text { take into consideration for a colonic } \\
\text { wall voxel }\end{array}$ \\
\hline Convexity & $\mathrm{T}_{\text {convex }}$ & $\begin{array}{l}\text { Polyp } \\
\text { candidate } \\
\text { generation }\end{array}$ & $\begin{array}{l}\text { Distance between a neighbor point and } \\
\text { the intersection point of the local } \\
\text { gradient with the tangent plane }\end{array}$ \\
\hline $\begin{array}{l}\text { Hits/total } \\
\text { ratio }\end{array}$ & $\mathrm{T}_{\text {hits }}$ & $\begin{array}{l}\text { Polyp } \\
\text { candidate } \\
\text { generation }\end{array}$ & $\begin{array}{l}\text { Minimum number of neighbors relative } \\
\text { to the total number of neighbors that } \\
\text { have to satisfy the convexity threshold }\end{array}$ \\
\hline $\begin{array}{l}\text { Sphere } \\
\text { radius }\end{array}$ & $\mathrm{T}_{\text {radius }}$ & $\begin{array}{l}\text { Polyp center } \\
\text { generation }\end{array}$ & $\begin{array}{l}\text { Distance between the current point and } \\
\text { the center of an imaginary sphere }\end{array}$ \\
\hline Extraction & $\mathrm{T}_{\text {extract }}$ & $\begin{array}{l}\text { Polyp } \\
\text { extraction }\end{array}$ & $\begin{array}{l}\text { Minimal value in the center map to be } \\
\text { considered as polyp candidate }\end{array}$ \\
\hline
\end{tabular}

Table 1. Threshold parameters, their name, symbol, appearance and meaning.

\subsection{Segmentation}

The goal of segmentation is to extract the colonic wall, in order to apply further processing on it. This step will reduce the amount of voxels we take into account as being candidates.

Our segmentation is based on a classical region-growing algorithm, as described in [10]. The user chooses a seed voxel and a region of connected voxels meeting the threshold criteria $\left(\mathrm{T}_{\text {segment }}\right)$ is grown outwards in a breadth first manner. $\mathrm{T}_{\text {segment }}$ can be adjusted based on the intensity values of the structure of interest. Sometimes a simple region growing operation may not be able to fill the entire desired structure (an air filled colon with a collapsed segment or due to colonic fluid). Multiple seed points can be specified and then multiple regions are grown separately. Region growing will detect voxels (the set A) given by colonic air. Colonic wall is assumed to be the set $\mathrm{C}$ of all adjacent voxels to $A$, and having a value higher than $T_{\text {segment }}$. For each voxel 
belonging to A all its 26 neighbors are taken into consideration, this can lead to a wall thickness of 2 voxels.

The obtained result $\mathrm{C}$ is a subset of the colonic wall because in some patients where wet preparation was used, the air-fluid boundary was also classified as colonic wall, and the submerged parts of the colonic wall were not segmented. A solution to this problem is the use of digital cleansing prior to CAD [2].

\subsection{Polyp Candidates Generation}

Only a small number of elements belonging to $\mathrm{C}$ will turn into polyp candidates. At this step the main criteria for polyp candidate selection is the convexity or the concavity of the wall over a region of interest.

To find possible candidates for each voxel in $\mathrm{C}$ we compute its gradient. The gradient will be perpendicular to the wall and oriented from air towards tissue. A Zucker and Hummel operator (an extension of the two-dimensional gradient to three dimensions) [10] is applied. Basically this operator will give as output the surface normal in the considered point. Having the gradient and the current point we can find the equation of the plane tangent to the surface in the current point.

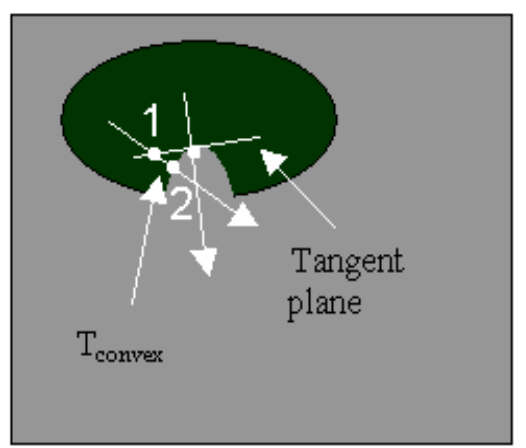

(a)

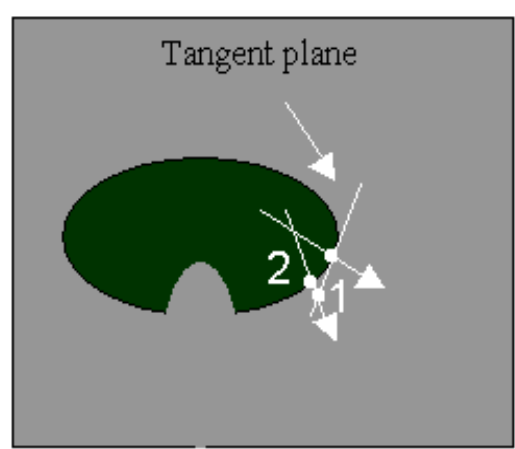

(b)

Fig. 2. Difference between convex and concave surfaces; notice the different relative positioning of point 1 and point 2. For convex surfaces (a) point 1 is situated in negative direction relative to point 2 , while for concave surfaces (b) point 1 is situated in positive direction relative to point 2 on the line given by the local gradient. For convex surfaces we apply a threshold based on $\mathrm{T}_{\text {convex }}$.

Suppose $v \in C, B_{v}$ is the bounding box around $v$ defined by $T_{b o x}$ and $P_{v}$ is the tangent plane in $\mathrm{v}$. Then for each $\mathrm{w} \in \mathrm{B}_{\mathrm{v}} \cap \mathrm{C}$ we compute its gradient and the intersection of this gradient with $P_{v}$, say $i_{v w}$. The relative location of $i_{v w}$ with regards to $\mathrm{W}$ can be exploited to assess convexity or concavity of the colon's surface in $\mathrm{v}$. Furthermore if the surface is convex, $T_{\text {convex }}$ can be applied to the distance $d$ between $\mathrm{w}$ and $\mathrm{i}_{\mathrm{vw}}$.

For each $v \in C$ two values $\left(V_{c}, V_{t}\right)$ are computed. $V_{c}$ is the number of voxels situated in $B_{v} \cap C$ that satisfy $T_{\text {convex }}$, while $V_{t}$ is the total number of voxels in $B_{v} \cap C$. All the voxels $\mathrm{v}$ for which $\mathrm{V}_{\mathrm{c}} / \mathrm{V}_{\mathrm{t}}$ is higher than $\mathrm{T}_{\text {hits }}$ are declared candidates. 

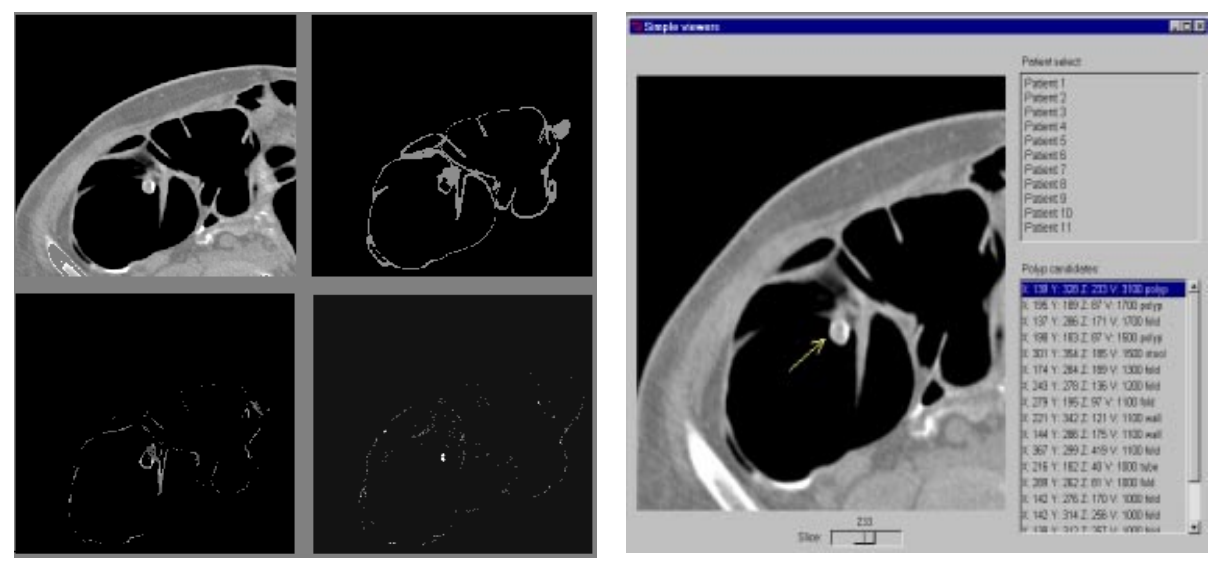

Fig. 3. The figure illustrates the four steps of our algorithm. Although our approach is truly 3D, we only show results on $2 \mathrm{D}$ slices. On the left a $2 \mathrm{D}$ region of interest is presented first as an original slice, then the results of segmentation, polyp candidate generation and polyp center generation are shown. On the right the user interface of the polyp presentation module is captured.

Due to the fact that haustral folds and polyps have similar convexity properties, this step will not only generate voxels that belong to polyps, but additionally voxels situated on haustral folds, and some voxels caused by irregularities on the colonic wall. Discrimination between voxels belonging to real polyps and those belonging to haustral folds is therefore a next step.

\subsection{Polyp Center Generation}

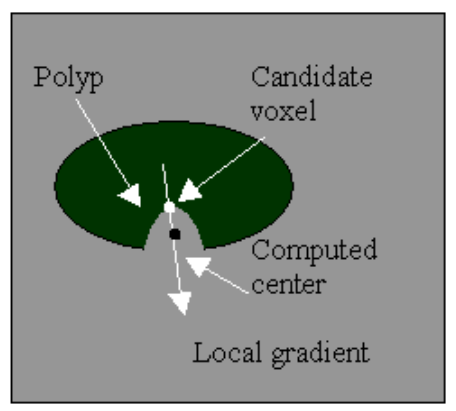

Fig. 4. Simplified illustration (2D example) of how the sphere centers are computed.
This discrimination is based on the fact that folds have a fairly cylindrical shape while polyps have mainly a spherical shape.

First for all of the previously selected candidates we compute a sphere center. The sphere has the point on its surface and the local gradient perpendicular to its surface. The radius $\left(\mathrm{T}_{\text {radius }}\right)$ of the sphere can be selected and has to be in close correspondence with the size of the polyps to be detected. Because folds have a cylindrical shape the centers given by such structures will be dispersed along a line. For polyps however these centers will converge towards a relatively small area. For polyps having the shape of a perfect sphere (semi-sphere) these centers would converge towards a single point. 

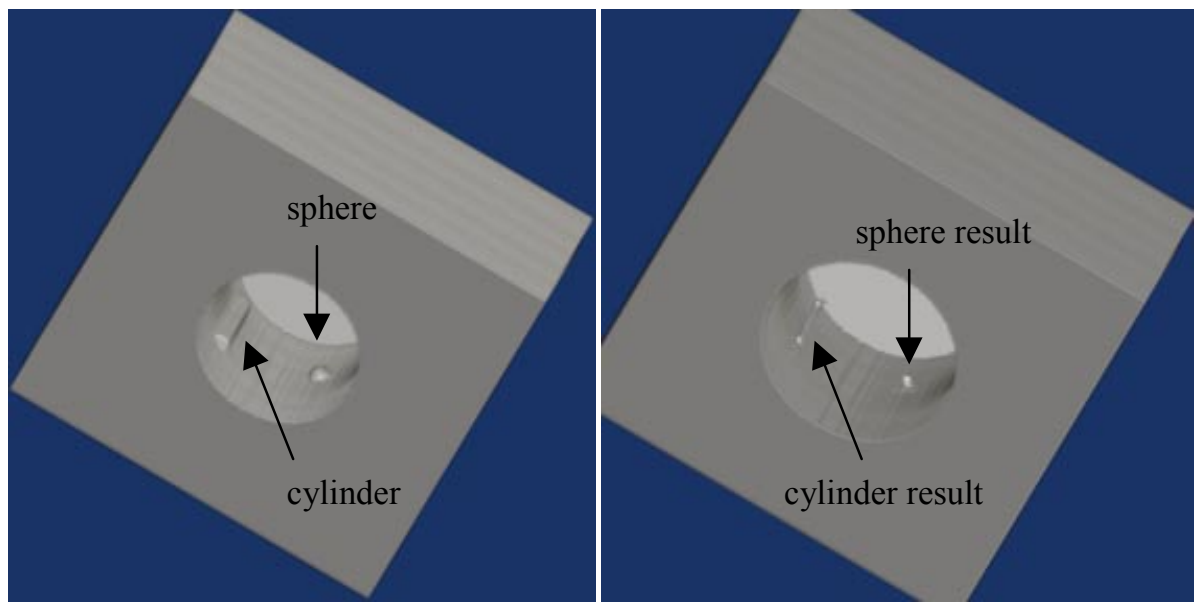

Fig. 5. The dispersion of centers for a cylinder, as opposed to a sphere. A 3D rendering of the original phantom (left) and of the results (right) are presented.

\subsection{Polyp Extraction and Presentation}

The previous step generates a map that represents the concentration of sphere centers in the data volume. As we want to present to the user polyp candidates on axial slices, given this map, we have to extract local maxima. To do this we look for values

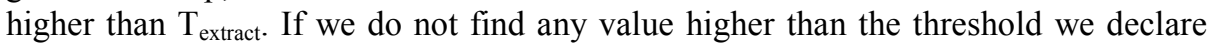
the patient as negative. When searching for local maxima we have to take into account that centers are concentrated in small areas, so for each of these areas only one response is wanted. To solve this problem a non-maximum suppression technique is applied.

Finally, the local maxima thus obtained are pointed out to the reading radiologist as polyp candidates.

\subsection{Initial Threshold Values Determination}

To find initial values for thresholds $\left(T_{\text {box }}, T_{\text {convex }}, T_{\text {hits }}, T_{\text {extract }}\right)$, we applied our method on a software phantom containing a perfect cylinder and a semi-sphere with a diameter of $10 \mathrm{~mm}$, similar to the polyps of interest. The cylinder was mimicking a haustral fold while the semi-sphere was considered to be perfect polyp. Also we used this phantom to verify our presumption that on haustral folds centers will disperse whereas for polyps they will converge towards small regions (see Figure 5).

\section{Results}

The presented CAD method was applied to 18 different CT-volumes, belonging to eighteen patients that underwent both CT-colonography and standard colonoscopy. 
Informed consent was obtained from all patients. CT was performed using a Multi Slice Helical CT (Siemens, Volume Zoom) using a 4x1 mm detector configuration, 7 $\mathrm{mm}$ table feet per $0.5 \mathrm{~s}$ tube rotation, $0.8 \mathrm{~mm}$ reconstruction increment as well as 60 effective mAs and $120 \mathrm{kV}$. The average number of slices was 496.

Colonoscopy was taken as ground truth; results from CAD were compared with the results obtained by a radiologist using the CTC method of axial-3D [2]. Nine of the patients had polyps proven by colonoscopy, while the other nine were declared negative. The size of the polyps was ranging from 3 up to $20 \mathrm{~mm}$ and the total number of polyps was 20 ( 1 of $3 \mathrm{~mm}, 3$ of $5 \mathrm{~mm}, 2$ of $6 \mathrm{~mm}, 9$ of $10 \mathrm{~mm}, 4$ of $10 \mathrm{~mm}$ flat lesions, and 1 of $20 \mathrm{~mm}$ ) (Table 2).

Our method detected all the nine polyps of $10 \mathrm{~mm}$ or larger, 1 from 2 polyps of $6 \mathrm{~mm}, 1$ from 3 polyps of $5 \mathrm{~mm}$ and missed the polyp of $3 \mathrm{~mm}$ and the four flat lesions of $10 \mathrm{~mm}$. The average computation time was 23.30 minutes, on an Intel Pentium III system running at $533 \mathrm{MHz}$ and having $512 \mathrm{MB}$ of RAM. The total number of false positives was 147 , which gives us a mean value of 8.16 false positives per case. Additionally we can mention that an average of 3.22 seed points was needed to segment correctly the colonic wall. The causes of false positives were colonic wall $(45.58 \%)$, haustral folds $(31.97 \%)$, colonic stool or fluid $(12.25 \%)$, the insuflation tube $(7.48 \%)$, and the ileocecal valve $(2.72 \%)$.

\begin{tabular}{|l|r|r|r|}
\hline Polyps & Total & Detected & Sensitivity \\
\hline Smaller than 5mm & 1 & 0 & $0 \%$ \\
\hline Between 5-9 mm & 5 & 2 & $40 \%$ \\
\hline Flat lesions & 4 & 0 & $0 \%$ \\
\hline Bigger than 10mm & 10 & 10 & $100 \%$ \\
\hline Overall & 20 & 12 & $60 \%$ \\
\hline
\end{tabular}

Table 2. Total number of polyps, detected number of polyps and sensitivity overall and for each group of polyps.

\section{Discussion and Conclusions}

Although the size of significant polyp is a debate subject between different radiologists and also between radiologists and gastroenterologists, we assumed that $10 \mathrm{~mm}$ polyps can be accepted as significant one's for virtual colonography. Therefore our primary goal was the detection of polyps bigger than $10 \mathrm{~mm}$. A secondary goal was a reasonable number of false positives per case. Using the polyp presentation application the radiologist can quickly go through the polyp candidate list and discriminate between real and false positive findings.

From literature, we can deduce that there is a strong correlation between sensitivity and the number of false positives generated. For example Tomasi et al. [6] are using a graph method optimized for the detection of polyps larger than $10 \mathrm{~mm}$. They achieve a sensitivity of $100 \%$ with a number of false positives (FP) as high as 50 per data set. Yoshida et al. [7] reported a sensitivity of $90 \%$ for polyps between $7-12 \mathrm{~mm}$ and $1 \mathrm{FP}$ per case. To achieve a sensitivity of $100 \%$ they reported an increase towards $1.5 \mathrm{FP}$ per case. Beaulieu et al. [5] developed three different methods for polyp detection. Their contour normal method has a sensitivity of $96.4 \%$ for polyps larger than $5 \mathrm{~mm}$ at the cost of 25 FP per data set. The sphere fit method returns a number of $47 \mathrm{FP}$, 
meanwhile the surface curvature method has a low number of FP only 4, but a decreased sensitivity as well (3 out of 7 polyps detected).

We have to stress that we are looking at CAD as a prospective tool and not as a retrospective one. We used the same parameter settings for all of our patients, positives or negatives. However if we would count only the number of false positives until all the polyps bigger than $10 \mathrm{~mm}$ have been identified, then we would only have $17 \mathrm{FP}$ findings in 9 cases, so an average of $1.88 \mathrm{FP}$ per case comparable with results given by other authors. Of course this kind of evaluation can be done only for retrospective studies.

The results of our experiments are at least encouraging. CAD will probably become the most common way of doing CTC, improving on current accuracy, efficiency and costs. Many further improvements to our algorithm are possible:

1. Application of digital cleansing prior to $\mathrm{CAD}$, in order to remove the colonic fluid and thus eliminating the necessity of multiple seed points

2. Introduction of a polyp database when labeling a polyp candidate and taking the decision based on previous knowledge.

3. From the implementation point of view changing our data structures will lead to a better balance between speed and memory requirements.

\section{Acknowledgement}

This work is part of the GOA/99/05 project: "Variability in Human Shape and Speech", financed by the Research Fund, K.U. Leuven, BELGIUM.

\section{References}

1. Johnson C.D. (2000) CT Colonography, Mayo Clinic Experience, Second International Symposium on Virtual Colonoscopy, Boston proceedings, October 2000, pp 16-18

2. Kiss G. et al. (2001) An Image Processing and Visualization System for Virtual Colonography, Technical Report: KUL/ESAT/PSI/0103, KU Leuven Belgium, June 2001

3. Nappi J., Dean P.B. (2000) A multiscale algorithm for segmenting calcifications from high-resolution mammographic specimen radiographs, J Digit Imaging, May 2000, 13(2 Suppl1):130-2

4. Ukai et al. (2000) Computer Aided Diagnosis System for Lung Cancer Based on Retrospective Helical CT Images, CARS proceedings, June 2000, pp 767-772

5. Beaulieu C.F. (2000) Computer Aided Detection of Colonic Polyps, Second International Symposium on Virtual Colonoscopy, Boston proceedings, October 2000, pp 73-77

6. Tomasi C., Gokturk S.B. (2000) A Graph Method for the Conservative Detection of Polyps in the Colon, Second International Symposium on Virtual Colonoscopy, Boston proceedings, October 2000, pp 105

7. Yoshida H. et al. (2000) Computer Aided Detection of Colonic Polyps in CT Colonography, Second International Symposium on Virtual Colonoscopy, Boston proceedings, October 2000, pp 104

8. Paik D.S. et al. (2000) Computer Aided Detection of Polyps in CT Colonography: Free Response ROC Evaluation of Performance, RSNA, Chicago, 2000.

9. Summers R.M. et al. (2000) Automated polyp detector in CT colonography: feasibility study, Radiology 2000 Jul;216(1):284-90

10. Ballard D.M., Brown C.M. (1982) Computer Vision, Prentice-Hall, pp 149-166 